\title{
Overtraining, extinction, and reversal learning by kittens
}

\author{
J. M. WARREN \\ The Pennsylvania State University, University Park, Pennsylvania 16802
}

\begin{abstract}
Two groups of kittens received 0 or 112 overtraining trials after learning a brightness discrimination. Both groups underwent extinction of differential choice responses and then learned the reversed brightness discrimination. The overtrained and nonovertrained animals did not differ significantly in rate of reversal learning, and both groups showed a significant preference for the old positive stimulus when differential reinforcement was reinstated in reversal training. According to Sutherland and Mackintosh, these results show that kittens lack stable attention and should be inept in dealing with reversal and probability problems. This is not the case, raising doubts about the adequacy of their account of interspecies differences in learning by vertebrates.
\end{abstract}

According to Sutherland and Mackintosh's (1971) model, two processes are involved in discrimination learning by animals. The subject must first learn to switch in the analyzer which detects differences between the reinforced and nonreinforced stimuli, and then learn to attach appropriate responses to the outputs of the relevant analyzer. In other. words, the animal must independently learn to attend to the relevant stimulus dimension, and to associate reward and nonreward with specific values on that dimension.

The relation between amount of discrimination learning and relative strength of analyzers and response attachments differs across species. In rats, response attachments reach asymptotic levels more rapidly than do analyzers. Overtraining beyond criterion affects primarily the degree to which the relevant analyzer is switched in, since response attachments are already at nearly asymptotic strength when a rat reaches criterion on a discrimination problem. This model accounts for two conspicuous differences in reversal leaming between groups of rats which were or were not overtrained after learning an initial discrimination: the overtraining reversal effect (ORE), and the "dip" effect.

Overtraining, under conditions specified by Mackintush $(1969 \mathrm{~b})$, facilitates the reversal of visual discriminations by rats. The superior performance of the overtrained rats is presumed to reflect their continued responding to the relevant dimension after extinction of their original response attachments in reversal learning. Slower reversal learning by nonovertrained rats is attributed to these animals extinguishing responses to the appropriate analyzer and switching to inappropriate analyzers like position.

The dip effect is observed when overtrained and nonovertrained groups of rats are trained on a visual discrimination and then tested in extinction until they choose the originally rewarded and nonrewarded stimuli equally often, before training on the reversed discrimination with differential reinforcement. In reversal learning, overtrained rats show an orderly

This research was supported by Grant $\mathrm{MH} 04726$ from the National Institute of Mental Health, U.S. Public Health Service. increase in the number of correct responses from chance to criterion. Nonovertrained rats, however, show a marked preference for the old positive stimulus upon the reinstatement of differential reinforcement in reversal training; their learning curves dip to levels significantly below chance.

This pattern of results suggests that the extinction trials given between discrimination and reversal training have different effects on analyzer and response attachment strengths in the two groups of rats. According to Sutherland and Mackintosh (1971), extinction weakens response attachments more than the analyzer in the overtrained group, and weakens the analyzer more than response attachments in the nonovertrained groups, since only nonovertrained rats persevere in responding to the initially rewarded stimulus in reversal learning.

Quite different results are obtained in similar experiments with birds and fish. Birds rarely and fish never show the ORE. Comparisons between overtrained and nonovertrained chicks yield dip effects that are attenuated or reversed relative to those seen in rats (Mackintosh, 1965). Preference tests given to goldfish after extinction of a discrimination habit reveal no difference between overtrained and nonovertrained subjects; both groups behave like nonovertrained rats (Mackintosh, Mackintosh, Safriel-Jorne, \& Sutherland, 1966).

Sutherland and Mackintosh (1971) claim the evidence indicates that in rats "analyzers are strengthened and weakened slowly relative to responses, in lower birds, rather more rapidly, in fish, much more rapidly" (p. 442). This conclusion leads them to assert that attention in birds is less stable, and in fish, much less stable, than in rats. It follows, on their theory, that birds should be somewhat, and fish markedly, inferior to rats on learning tasks that. require stable attention in the face of inconsistent reinforcement contingencies. Evidence confirming this prediction has been obtained in studies of reversal and probahility learning (Mackintosh, 1969a).

Overtraining does not lacilitate reversal learning by cats reversed after overtraining on a brightness 
Table 1

Mean Errors and Trials to Criterion for Overtrained (OT) and Control (C) Groups

\begin{tabular}{lccrrr}
\hline & \multicolumn{2}{c}{ Errors } & & \multicolumn{2}{c}{ Trials } \\
\cline { 2 - 3 } \cline { 5 - 6 } Task & OT & C & & OT & C \\
Discrimination & 58 & 51 & & 162 & 146 \\
Overtraining & 8 & & & 112 & \\
Extinction & & & 97 & 62 \\
Reversal & 96 & 82 & & 200 & 179 \\
\hline
\end{tabular}

discrimination (Hirayoshi \& Warren, 1967). This experiment largely replicated Hirayoshi and Warren's procedure, except that reversal training was preceded by a phase during which the choice responses acquired in initial learning were extinguished. The major purpose of the present experiment was to determine if nonovertrained cats do, and overtrained cats do not, show the dip effect as is the case with rats. It also afforded an opportunity to assess the reliability of Hirayoshi and Warren's (1967) finding does not accelerate reversal learning by cats.

\section{METHOD}

\section{Subjects}

Twenty-six experimentally naive kittens were tested under $23 \mathrm{~h}$ of food deprivation and rewarded with $3 \mathrm{~g}$ of pork kidney for correct responses on differentially rewarded trials. Fourteen overtrained (OT) kittens were aged 60 days at the start of the experiment; 12 litter mate controls (C) were started as soon as the OT animals with which they were matched completed 10 days of discrimination training. This procedure was used to insure that the OT and $C$ groups would be the same age at the start of extinction and reversal training, and that the same interval elapsed between discrimination and extinction testing.

\section{Apparatus and Trial Procedure}

The apparatus was a modified Grice box, $36 \mathrm{~cm}$ high and painted gray throughout. The $27.5 \times 55 \mathrm{~cm}$ startbox, with opaque and transparent guillotine doors, opened into a $40-\mathrm{cm}$ deep choice area, beyond which were two $30 \times 42.5 \mathrm{~cm}$ response compartments separated by an opaque wall. Four pairs of stimuli differed in brigh tness, shape, and size: (a) small $(36 \mathrm{sq} \mathrm{cm}$ ) white triangle and large $(81 \mathrm{sq} \mathrm{cm})$ black circle; (b) small black triangle and large white circle; (c) large white triangle and small black circle; (d) large black triangle and small white circle. The incentive was concealed behind the correct stimulus and the position of the rewarded object was varied from trial to trial in a balanced irregular sequence.

A trial began when the experimenter removed the opaque door of the startbox. The transparent door was raised $3 \mathrm{sec}$ later, and the kitten was allowed to enter one of the response compartments. If the subject chose correctly, it was allowed to approach and eat the reward; if it responded incorrectly, it was permitted to approach the unrewarded stimulus but was prevented from correcting its error. The intertrial interval was approximately $10 \mathrm{sec}$.

\section{Procedure}

Adaptation. The animals were tested in the apparatus with no stimulus objects but with meat visible in both response compartments until they completed 16 reinforced free choice trials within $15 \mathrm{~min}$. Most subjects satisfied this criterion in a single test session.
Preference Test. Each of the four pairs of stimuli was presented for four trials in a balanced irregular sequence, and all responses were reinforced. The position and object chosen on each trial was recorded to determine the subjects' stimulus preference prior to the introduction of differential reinforcement.

Brightness Discrimination Training. The four stimulus pairs were each presented on four trials per day in a balanced irregular sequence. All of the kittens were required to choose consistently the brightness value which was not preferred in the previous preference test, without reference to irrelevant variations in the size, shape, and position of the rewarded stimulus. In both discrimination and reversal training, the criterion of mastery was 15 correct responses in a daily session of 16 trials.

Overtraining. The OT group was tested on 16 trials per day for 7 days after reaching criterion on the brightness discrimination task, for a total of 112 overtraining trials.

Extinction. Neither stimulus object was reinforced in this phase, during which the animals were tested on 16 trials per day until they responded to the white and black objects on five trials each, over a set of 10 consecutive trials, within a single session.

Reversal. The stimulus-reward contingencies were now reversed with respect to original leaming and the kittens received 16 differentially rewarded trials per day until they made 15 correct responses in a single session.

\section{RESULTS}

\section{Preference Tests}

Few of the kittens responded to cues in any dimension significantly more often than chance (at least 12 of 16 trials, $p<.04$ ). For the 26 subjects, the frequency of significant preferences on the four sorts of cues was: position, 9 ; brightness, 2 ; form, 1 ; and size, 0 . The $\mathrm{C}$ and OT groups did not differ significantly in respect to the number of cats showing significant preferences on any cue dimension.

\section{Learning}

The results are summarized in Table 1 and Figure 1. Table 1 gives the mean number of trials and errors to criterion in discrimination and reversal learning by the OT and $\mathrm{C}$ groups, the mean number of errors by the OT group during overtraining, and the number of trials both groups required to meet the extinction criterion. U-tests indicate that the groups did not differ significantly in discrimination, extinction, or reversal performance.

Reversal learning curves for the OT and $\mathrm{C}$ groups are presented in Figure 1, which shows that both groups performed at below chance levels on the first four days of reversal training. The performance of Group $C$ was significantly below chance on Day $1 \quad\left(\mathrm{X}^{2}=22.25\right.$, $p<.05$ with $11 \mathrm{df}$ ) and the scores of the OT subjects were significantly lower than chance on both Day 1 $\left(\mathrm{X}^{2}=33.13, \mathrm{p}<.01\right.$ with $\left.13 \mathrm{df}\right)$ and Day 2 $\left(X^{2}=22.88, p<.05\right)$.

I am not aware of any direct determination of the effects of extinguishing choice responses upon subsequent reversal learning. It seems, therefore, worthwhile to present Figure 2 which compares the results of this experiment with those obtained from cats which learned a reversal problem under almost identical 
conditions, but without any extinction training interposed between discrimination and reversal learning (Hirayoshi \& Warrer, 1968). This figure shows the mean number of trials to successive criteria of $1,2,3, \ldots, 15$ correct responses in a single session. The data indicate that extinction training does not impair reversal learning, since both extinction groups are superior to the two groups reversed without extinction of initial choice responses. The curves also show that overtraining does not substantially facilitate any phase of reversal performance by cats, whether or not the animals are tested in extinction before reversal training.

\section{DISCUSSION}

Overtraining had no significant effect on either the rate or course of reversal leaming by the kittens studied in this experiment, even though they were trained under the conditions most favorable for the ORE in rats: on a discrimination between stimuli on a dimension initially low in attention value, with multiple irrelevant cues, and with large rewards (Mackintosh, 1969b). As in an earlier experiment (Hirayoshi \& Warren, 1967), no advantage in reversal learning accrued to the kittens overtrained on the initial discrimination.

Both overtrained and control kittens showed significant preferences for the old positive stimulus wher, after extinction, rewards were again provided in reversal training. This response pattern implies that both groups of cats switched out the appropriate analyzer

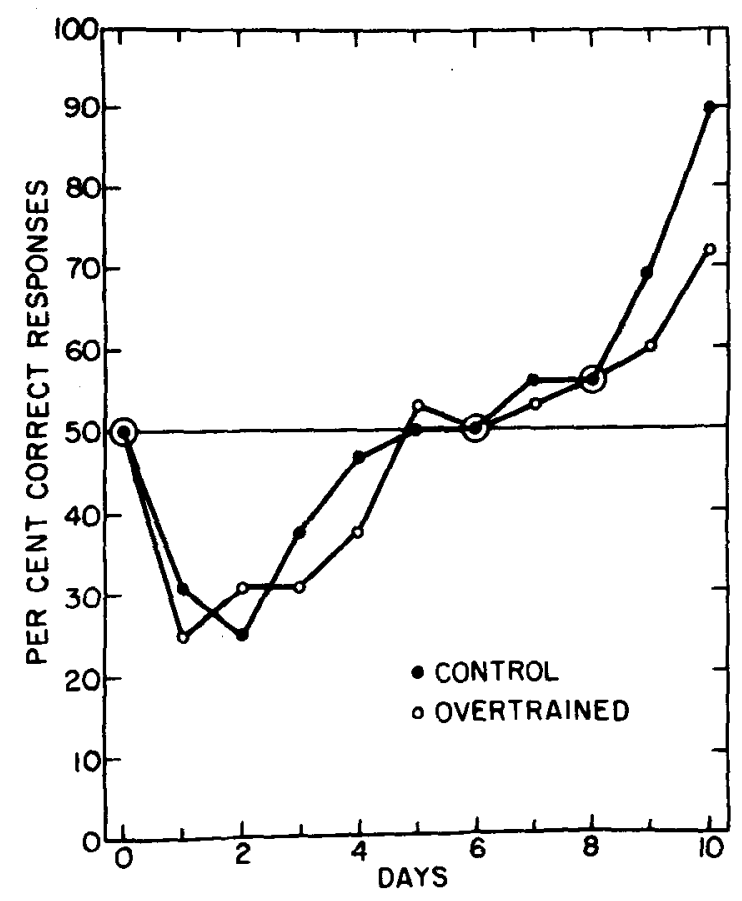

Figure 1. Reversal learning by overtrained and control kittens

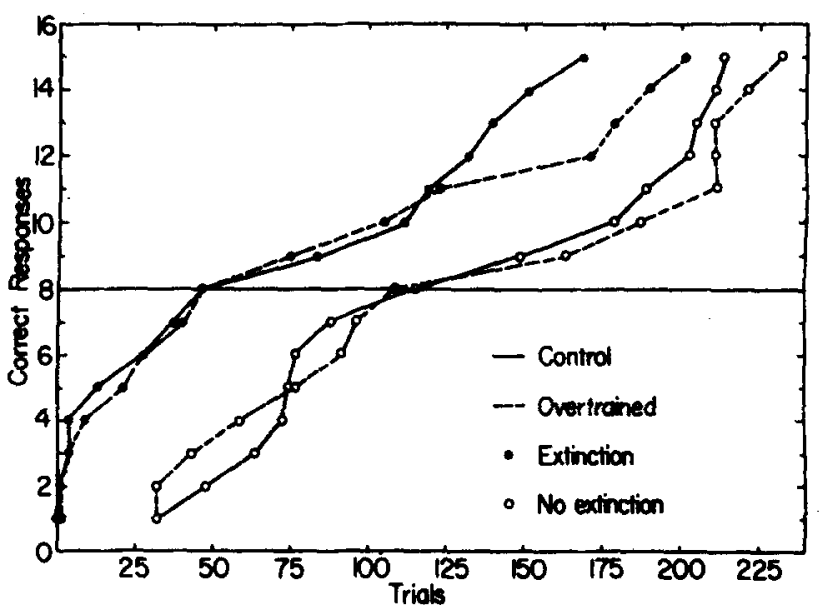

Figure 2. Reversal learning curves for cats trained to criterion or overtrained, which were then reversed immedintely or after extinction of differential choice responses.

rather than extinguishing response attachments during extinction testing. If this is so, cats must be classed with birds and fish as having unstable attention. Sutherland and Mackintosh (1971) would thus seem obliged to predict that cats, like birds and fish, should be poor at reversal and probability learning. But the experimental evidence (Schweikert \& Treichler, 1969; Warren, 1966) shows that this is not true; the performance of cats on reversal and probability learning tasks is essentially identical with that of rats, rhesus monkeys, and other mammals. Therefore, Sutherland and Mackintosh's explanation of the inferior performance of birds and fish on reversal and probability learning problems in terms of labile attention mechanisms must be regarded as an account of limited generality, which does not accurately describe discrimination leaming by cats.

\section{REFERENCES}

Hiray oshi, I., \& Warren, J. M. Overtxaining and reversal learning by experimentally naive kittens. Joumal of Comparative and Phydological Psycholoty, 1967, 64,507-510.

Mackintosh, N. J. Ovextraining, extinction and reversal in rets and chicks. Joumal of Comparative and Physiological Psychology, 1965, 59, 31-36.

Mackintosh, N. J. Comparative psychology of serial reversal and probability leaming: Rats, birds and fish. In R. Gibert and $N$. S. Sutherland (Dds.), Animal discrimination learning. London: Academic Press, 1969. Pp. 137-167. (a)

Mackintosh, N. J. Further analy ds of the overtraining revesal effect. Joumal of Comparative and Physiological Psycholocy, Monoxraph Supplement, 1969b, 67, 1-18.

Mackintosh, N. J.. Mackintosh, J., Safriel-Jorne, O., \& Sutherland, N. S. Overtraining, reversal and extinction in the goldtish. Animal Behaviour, 1966, 14, 314-318.

Schweikert, G. E., \& Treichler, F. R. Visual probability learning and reversal in the cat. Journal of Compantive and Phy aiological Psy chology, 1969, 67, 269-272.

Sutherland, N. S., \& Mackintosh, N. J. Mechaniems of animal discrimination learning. New York: Academic Press, 1971.

Warren. J. M. Reversal leaming and the formation of learning sets by cats and rhesus monkeys. Journal of Compartive and Physiological Psy choloty, 1966, 61, 421-428. 